\title{
Fast Dynamic Reconstruction Algorithm with Joint Bilateral Filtering for Perfusion C-arm CT
}

\author{
Michael Manhart, Markus Kowarschik, Andreas Fieselmann, Yu Deuerling-Zheng and Joachim Hornegger
}

\begin{abstract}
Tissue perfusion measurement using C-arm angiography systems is a novel technique with potential high benefit for catheter-guided treatment of stroke in the interventional suite. However, perfusion C-arm CT (PCCT) is challenging: the slow $\mathrm{C}$-arm rotation speed only allows measuring samples of contrast time attenuation curves (TACs) every $5-6 \mathrm{~s}$ if reconstruction algorithms for static data are used. Furthermore, the peaks of the tissue TACs typically lie in a range of 5 - $30 \mathrm{HU}$, thus perfusion imaging is very sensitive to noise. We present a dynamic, iterative reconstruction (DIR) approach to reconstruct TACs described by a weighted sum of linear spline functions. The optimization problem is solved using an appropriate initialization and a Landweber-based optimization strategy with a modified backprojection step. To reduce noise a novel regularization technique based on Joint Bilateral Filtering (JBF) is introduced. The algorithm is evaluated using simulation data created with a dynamic cylindrical phantom, a realistic digital brain phantom and real measured data from an animal study with a canine stroke model. Results indicate that the DIR algorithm qualitatively and quantitatively improves reconstructed TACs and perfusion maps compared to classical Feldkamp (FDK) reconstruction. For the brain phantom study the Pearson correlation (PC) of the reconstructed cerebral blood flow (CBF) maps to the ground truth increased from 0.82 (FDK) to 0.87 (DIR). For the canine study the PC of the CBF maps to coregistered perfusion $\mathrm{CT}$ maps increased from 0.61 (FDK) to 0.73 (DIR).
\end{abstract}

Index Terms-Perfusion imaging, dynamic reconstruction, Carm CT, stroke treatment

\section{INTRODUCTION}

Perfusion CT (PCT) is an important imaging modality for diagnosis in case of an ischemic stroke event [1]. Time attenuation curves (TACs) in tissue and vessels are extracted from a time series of brain volumes acquired after a contrast bolus injection. Perfusion parameter maps calculated from TACs, which represent quantities such as cerebral blood flow (CBF), cerebral blood volume (CBV), and mean transit time (MTT), provide information about the extent of the affected tissue. They can be used to identify potentially salvageable ischemic tissue that may be reperfused by catheter-guided stroke therapy procedures such as intra-arterial thrombolysis. For this purpose the patient is transported to an interventional suite equipped with a C-arm angiography system, where perfusion measurement is not yet available. Perfusion measurement using

M. Manhart, A. Fieselmann and J. Hornegger are with Pattern Recognition Lab, Department of Computer Science, Friedrich-Alexander-Universität Erlangen-Nürnberg, Martensstr. 3, 91058 Erlangen, Germany. M. Kowarschik and Y. Deuerling-Zheng are with Siemens AG, Angiography \& Interventional X-Ray Systems, Forchheim, Germany. A. Fieselmann and J. Hornegger are with Erlangen Graduate School in Advanced Optical Technologies (SAOT).

Email: michael.manhart@cs.fau.de
C-arm systems would allow assessing the perfusion parameters directly before and during the interventional procedure and thus help to determine treatment success and endpoint [2]. Current $\mathrm{C}$-arm systems typically require $\sim 4-5 \mathrm{~s}$ to acquire the X-ray projection images needed to reconstruct one volume and a pause of $\sim 1 \mathrm{~s}$ between two successive acquisitions, which limits the temporal sampling of the TACs and makes perfusion C-arm CT (PCCT) challenging.

Recently new approaches have been presented to overcome these problems: Fieselmann et al. [3] proposed a new scanning protocol combining interleaved scanning and partial reconstruction interpolation. While providing improved temporal sampling and high computational efficiency, multiple scanning sequences are required, which increases irradiation and contrast agent dose to the patient. Serowy et. al [4] and Neukirchen et al. [5] showed iterative model-based approaches, which reconstruct TACs described by a sum of weighted smooth temporal basis functions in order to keep the degree of freedom relatively low. Although these algorithms can be implemented similarly to classical algebraic reconstruction techniques (ART) [6], the use of basis functions with non-compact support introduces additional computationally expensive steps. In [7] Neukirchen presents a computationally fast, analytic approach for computing the basis weights by a weighted Feldkamp (FDK) [8] reconstruction. However, recent developments in iterative reconstruction techniques, employing total variation (TV) [9] or wavelet based [10] regularization for example, have shown to highly improve reconstruction results in case of noisy or undersampled data. Since the peaks of the TACs inside the brain tissue lie typically in a range of 5 $30 \mathrm{HU}$ perfusion imaging is highly sensitive to noise, which makes PCCT an interesting application for iterative algorithms.

This work introduces a dynamic, iterative reconstruction (DIR) algorithm which is based on the iterative parameter optimization algorithm by Neukirchen et al. [5]. In contrast to [5] we use linear spline basis functions with compact support for describing the reconstructed TACs to reduce the computational effort. Additionally we introduce a novel denosing strategy based on joint bilateral filtering (JBF) [11], [12]. The algorithm is implemented GPU-based and evaluated using simulation data created with a digital phantom describing multiple arterial and tissue TACs, with an extension of the realistic digital brain perfusion phantom by Riordan et al. [13] and real measured data from an animal study with a canine stroke model. The brain phantom data and tools are published online to improve the reproducibility of this and future studies [14]. 


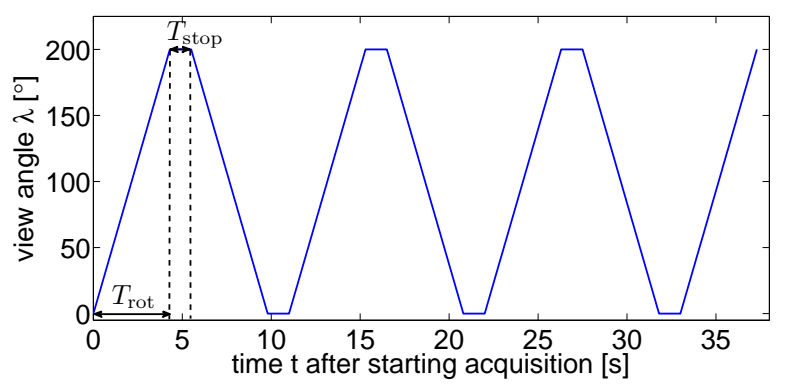

Figure 1: C-arm acquisition protocol

\begin{tabular}{cc}
\hline view-angle increment & $0.8^{\circ}$ \\
number of views per rotation $\left(N_{\text {proj }}\right)$ & 248 \\
angular range per rotation & $197.6^{\circ}$ \\
time per rotation $\left(T_{\text {rot }}\right)$ & $4.3 \mathrm{~s}$ \\
time between rotations $\left(T_{\text {stop }}\right)$ & $1.2 \mathrm{~s}$ \\
number of rotations $\left(N_{\text {rot }}\right)$ & 7 \\
total scanning time & $37.3 \mathrm{~s}$ \\
source-to-detector distance & $1200 \mathrm{~mm}$ \\
detector pixel size & $0.616 \times 0.616 \mathrm{~mm}^{2}$ \\
number of detector pixels $\left(N_{\mathrm{u}} \times N_{\mathrm{v}}\right)$ & $616 \times 480$ \\
& after $4 \times 4$ rebinning \\
total detector size & $\approx 380 \times 296 \mathrm{~mm}^{2}$ \\
\hline
\end{tabular}

Table I: Acquisition parameters

\section{ACQuisition Protocol}

This section describes the $\mathrm{C}$-arm perfusion acquisition protocol used for the simulation studies and real data acquisitions. Since currently available C-arm systems are not capable of continuous, uni-directional $\mathrm{C}$-arm rotations, the $\mathrm{C}$-arm is rotated in a bi-directional manner in forward and backward direction. At first one $\mathrm{C}$-arm rotation in forward and one in backward direction acquires baseline projections with the static anatomical structures. In each rotation $N_{\text {proj }}=248$ projections in an angular range of $197.6^{\circ}$ are acquired. After contrast agent injection the C-arm is rotated $N_{\text {rot }}=7$ times in bi-directional manner as shown in Figure 1. Each rotation takes $T_{\text {rot }}=4.3 \mathrm{~s}$ with a pause of $T_{\text {stop }}=1.2 \mathrm{~s}$ between each rotation. Thus direct reconstruction of the rotations would allow a temporal sampling of TACs with period $T_{\mathrm{s}}=T_{\text {rot }}+$ $T_{\text {stop }}=5.5 \mathrm{~s}$. The static projections are subtracted from the projections of the contrast-enhanced scans after logarithmic pre-processing, assuming that the patient head is not moved during acquisition. This generates the projection data vector $\mathbf{p}=\left[\mathbf{p}_{1}^{T} \cdots \mathbf{p}_{N_{P}}^{T}\right]^{T}, \mathbf{p} \in \mathbb{R}^{S_{\mathrm{P}} \cdot N_{\mathrm{P}}}$ containing only the pure contrast dynamics (and noise), where $S_{\mathrm{P}}=N_{\mathrm{u}} \cdot N_{\mathrm{v}}$ denotes the size of the detector in pixels with $N_{\mathrm{u}}=616$ columns and $N_{\mathrm{v}}=480$ rows and $N_{\mathrm{P}}=N_{\text {rot }} \cdot N_{\text {proj }}$ the total number of acquired contrast-enhanced projections. Furthermore the vector $\mathbf{t}^{\mathrm{P}}=\left[t_{1}^{\mathrm{P}} \cdots t_{N_{\mathrm{P}}}^{\mathrm{P}}\right]$ describes the acquisition time points of every projection in $\mathbf{p}$. Table I shows an overview over all acquisition parameters.

\section{Dynamic Reconstruction Algorithm}

\section{A. FDK}

For initialization and comparison standard FDK reconstruction [8] with Parker short-scan weights [15] is used. The filtering step applies a Shepp-Logan filter kernel [16] multiplied with a Gaussian of variance $\sigma_{\mathrm{K}}^{2}$ controlling smoothness and noise level in the reconstructed volumes.

\section{B. Dynamic Iterative Reconstruction (DIR)}

Mathematical Formulation: Since there is a continuous contrast flow during the acquisition, each of the $N_{\mathrm{P}}$ projections is taken from a different volume. Thus for an exact solution, we would have to reconstruct the $4 \mathrm{D}$ volume vector $\mathbf{x}=\left[\mathbf{x}_{1}^{T} \cdots \mathbf{x}_{N_{\mathrm{P}}}^{T}\right]^{T}, \mathbf{x}_{i} \in \mathbb{R}^{S_{\mathrm{v}}}$, consisting of $N_{\mathrm{P}} 3 \mathrm{D}$ volumes $X_{i} \in \mathbb{R}^{N_{\mathrm{x}} \times N_{\mathrm{y}} \times N_{\mathrm{z}}}, i=1 \ldots N_{\mathrm{P}}$ represented as a column vector $\mathbf{x}_{i} \in \mathbb{R}^{S_{\mathrm{v}}}, S_{\mathrm{V}}=N_{\mathrm{x}} \cdot N_{\mathrm{y}} \cdot N_{\mathrm{z}}$, where each voxel in a volume $X_{i}$ represents a sample of a reconstructed TAC. To describe the mapping of the $4 \mathrm{D}$ volume to the projection data, we define the system matrix $A$ assembled from matrices $A_{i}$ mapping the $3 \mathrm{D}$ volumes to the projection line integrals according to the acquisition geometry, such that $\mathbf{p}=A \mathbf{x}$ :

$$
A=\left(\begin{array}{cccc}
A_{1} & \mathbf{0} & \cdots & \mathbf{0} \\
\mathbf{0} & A_{2} & \ddots & \mathbf{0} \\
\vdots & \vdots & \ddots & \vdots \\
\mathbf{0} & \mathbf{0} & \cdots & A_{N_{\mathrm{P}}}
\end{array}\right) \text { with } \quad \begin{gathered}
A_{i} \in \mathbb{R}^{S_{\mathrm{P}} \times S_{\mathrm{V}}} \\
\quad A \in \mathbb{R}^{\left(N_{\mathrm{P}} \cdot S_{\mathrm{P}}\right) \times\left(N_{\mathrm{P}} \cdot S_{\mathrm{V}}\right)} .
\end{gathered}
$$

Of course, directly solving $A \mathbf{x}=\mathbf{p}$ for the exact solution $\mathbf{x}$ is not possible since the equation system is heavily underdetermined. Therefore we constrain the TACs described by $\mathrm{x}$ to be inside the subspace spanned by asymmetric linear spline functions, such that $\mathbf{x}=B \mathbf{w}$ with $B \in \mathbb{R}^{\left(N_{\mathrm{P}} \cdot S_{\mathrm{v}}\right) \times\left(N_{\mathrm{w}} \cdot S_{\mathrm{v}}\right)}$, and solve for the spline weights $\mathbf{w}=\left[\mathbf{w}_{1}^{T} \cdots \mathbf{w}_{N_{\mathrm{w}}}^{T}\right]^{T}, \mathbf{w}_{j} \in \mathbb{R}^{S_{\mathrm{v}}}$. The basis system is shown in Figure 2. The number of spline basis functions is $N_{\mathrm{w}}=2 \cdot N_{\mathrm{rot}}$, and the weight vectors $\mathbf{w}_{j}$ describe the contrast attenuation at the time points $t_{j}^{\mathrm{w}}$, where:

$$
t_{j}^{\mathrm{w}}= \begin{cases}\left\lfloor\frac{j-1}{2}\right\rfloor \cdot\left(T_{\text {stop }}+T_{\text {rot }}\right)+0.25 \cdot T_{\text {rot }} & j \text { odd } \\ \left\lfloor\frac{j-1}{2}\right\rfloor \cdot\left(T_{\text {stop }}+T_{\text {rot }}\right)+0.75 \cdot T_{\text {rot }} & j \text { even. }\end{cases}
$$

Thus $t_{j}^{\mathrm{w}}$ describes the temporal position of the knot of the linear spline belonging to the weight vector $\mathbf{w}_{j}$. The basis matrix $B$ computes the volume vectors $\mathbf{x}_{i}$, which describe the estimated contrast attenuation at time $t_{i}^{\mathrm{P}}$, by linear interpolation between the two closest weight vectors:

$$
\begin{array}{ll}
\mathbf{x}_{i}=\quad & \left(1-w_{i}\right) \mathbf{w}_{p}+w_{i} \mathbf{w}_{n} \text { with } w_{i}=\frac{t_{i}^{\mathrm{P}}-t_{p}^{\mathrm{w}}}{t_{n}^{\mathrm{w}}-t_{p}^{\mathrm{w}}} \\
\text { and } \quad & p=\max \left\{p \mid t_{p}^{\mathrm{w}}<t_{i}^{\mathrm{P}}, p=1 \ldots N_{\mathrm{w}}\right\}, \\
& n=\min \left\{n \mid t_{n}^{\mathrm{w}} \geq t_{i}^{\mathrm{P}}, n=1 \ldots N_{\mathrm{w}}\right\} .
\end{array}
$$

Exceptions need to be defined for the begin and for the end of the acquisition: if $0 \leq t_{i}^{\mathrm{P}} \leq t_{1}^{\mathrm{W}}$ then $\mathbf{x}_{i}=\frac{t_{i}^{\mathrm{P}}}{t_{1}^{\mathrm{w}}} \mathbf{w}_{1}$, and if $t_{i}^{\mathrm{P}}>t_{N_{\mathrm{w}}}^{\mathrm{w}}$ then $\mathbf{x}_{i}=\mathbf{w}_{N_{\mathrm{w}}}$. This reflects the assumption that we expect a rise of the contrast attenuation from $0 \mathrm{HU}$ in the beginning and a constant plateau phase of residual contrast in the end.

To reconstruct the basis weights $\mathbf{w}$ from the measured projection data $\mathbf{p}$, we solve the least-squares problem minimizing the Euclidean distance between the measured projection data $\mathbf{p}$ and the forward projected estimated $4 \mathrm{D}$ volume:

$$
\hat{\mathbf{w}}=\underset{\mathbf{w}}{\operatorname{argmin}}\|A B \mathbf{w}-\mathbf{p}\|_{2} .
$$




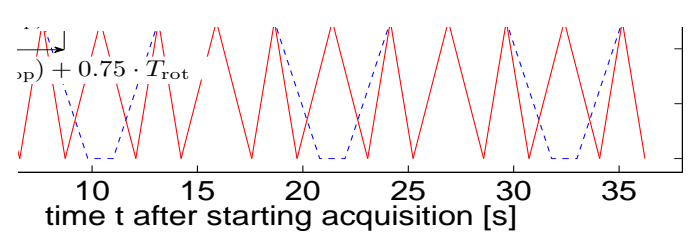

Figure 2: Basis functions for linear interpolation (red, solid) and relative angular $\mathrm{C}$-arm position (blue, dashed)

We solve this large scale problem as described in [5] by using a gradient-based iterative procedure based on an extended Landweber scheme. This results in a weight update step similar to classic ART-based algorithms:

$$
\mathbf{w}^{k+1}=\mathbf{w}^{k}+\beta \cdot B^{T} A^{T}\left(\mathbf{p}-A B \mathbf{w}^{k}\right) .
$$

The relaxation parameter $\beta$ controls the step size of the parameter update in each iteration, $A B$ describes a linear interpolation followed by forward projection and $B^{T} A^{T}$ is a weighted backprojection of the error image onto the basis weights.

Implementation: The computation of the weights $\mathbf{w}$ is done similarly as described in [5]. We reconstruct the 3D weight volumes $W_{j} \in \mathbb{R}^{N_{\mathrm{x}} \times N_{\mathrm{y}} \times N_{z}}, j=1 \ldots N_{\mathrm{w}}$, where each $W_{j}$ represents the weights in vector $\mathbf{w}_{j}$ as $3 \mathrm{D}$ volume, using a ray driven forward and a voxel driven back projector, both implemented in CUDA as described in [17]. To improve convergence speed, an ordered subset (OS) approach is used. The projections of each rotation are partitioned into 10 disjoint subsets maximizing the difference of the acquisition angle in each subset. In each iteration, the algorithm processes successively the projections of all rotations. For each projection $\mathbf{p}_{i}$ the corresponding volume $X_{i}$ is computed according to equation 2 using a GPU implemented linear interpolation, forward projected and subtracted with the measured projection image. The resulting error image is weighted with its associated basis function values $\left(1-w_{i}\right)$ and $w_{i}$, and back projected onto the corresponding weight volumes $W_{p}$ and $W_{n}$, respectively. After processing one subset of projections, all negative attenuation weights in the updated vector $\mathbf{w}$ are set to zero to ensure a physically correct solution.

Direct application of the optimization strategy of [5] in combination with linear basis functions converged slowly and the reconstructions were corrupted by streaking artifacts. Figure 3a shows a slice of the resulting temporal maximum intensity projection (MIP) volume of the TACs reconstructed from a digital brain phantom (see section IV-C for details) after 30 iterations, where severe streak artifacts around the high contrast vessel structures are visible. The MIP is created by taking the peak of the reconstructed TACs. Thus, a good initialization and a sophisticated optimization strategy are required such that the algorithm converges to a suitable result. Therefore all rotations are first reconstructed using the FDK

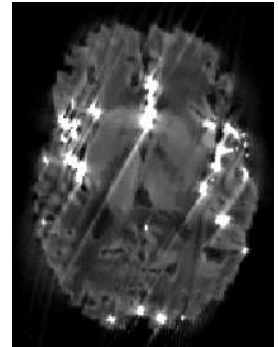

(a) Direct

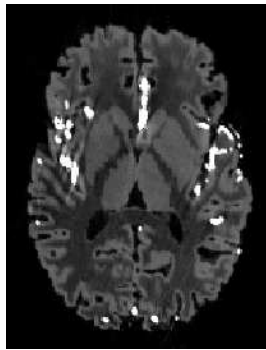

(b) Vessel masked

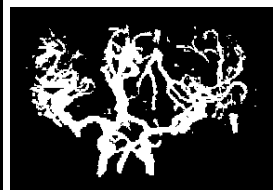

(c) Projection vessel mask
Figure 3: MIP reconstruction results [0 50] HU

algorithm. A sharp filter kernel is used to avoid blurring of high contrast vessels into the soft tissue. From the FDK reconstruction, initial TACs are calculated by linear interpolation, whereby each reconstructed rotation represents TAC samples at the temporal mid time point of its acquisition. The weight volumes $W_{j}$ are initialized using the interpolated TACs. To avoid the streak artifacts the back projection step is modified. Therefore the temporal MIP of the initial TACs is computed. By thresholding the MIP with threshold $\tau_{\text {MIP }}$ a vessel mask in volume space $V^{V}(\mathbf{v}): \mathbb{N}^{3} \rightarrow\{0,1\}$ is created, indicating which voxel $\mathbf{v}$ belongs to a vessel. Accordingly, vessel masks in projection space $V_{i}^{\mathrm{P}}(\mathbf{u}): \mathbb{N}^{2} \rightarrow\{0,1\}$ are computed for all $i=1 \ldots N_{\mathrm{P}}$ projections by a maximum intensity forward projection of $V^{\mathrm{V}}$. The projection vessel masks indicate which detector pixels $\mathbf{u}$ belong to a ray intersecting with a vessel structure (see Figure 3c). In all backprojection steps, pixels in the error image associated with a vessel intersecting ray by $V_{i}^{\mathrm{P}}$ are only backprojected onto voxels which belong to a vessel according to $V^{\mathrm{V}}$. This helps to avoid the severe streak artifacts as shown in the resulting MIP slice from the vessel masked reconstruction in figure $3 \mathrm{~b}$.

\section{Joint Bilateral Filtering}

Due to the high sensitivity of perfusion imaging to noise, a sophisticated regularization strategy for DIR allowing reliable reconstruction of TACs under noisy conditions is required. In the following we introduce a bilateral filter based denoising for regularization of the DIR. Bilateral filtering, introduced by [18] and made popular by [11], is a non-linear, edgepreserving noise filter using a combination of domain and range filtering. Mendrik et al. [19] presented a special adaption for PCT by replacing the range component by a time-intensity profile similarity (TIPS): each voxel of a 4D perfusion volume is replaced by a weighted combination of voxels of its corresponding temporally sampled 3D volume, which are spatially close and have high TIPS, i.e. the TACs the voxels belong to are similar. However, evaluating the TIPS measure between two voxels requires to calculate a sum of squared difference over the temporal dimension inducing a higher computational effort than standard 3D bilateral filtering. In our approach we only use the peak value of the TAC instead of incorporating the complete TAC to identify voxels belonging to similar structures (like vessels or healthy and stroke affected 


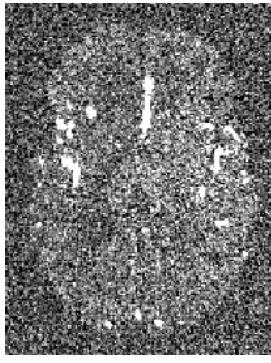

(a) Sharp FDK reconstruction

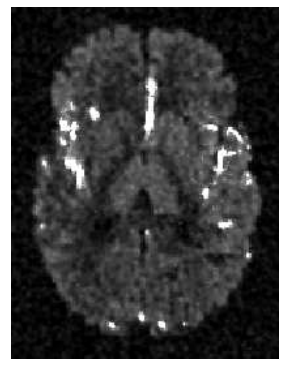

(b) Denoised FDK reconstruction
Figure 4: MIP noise reduction [0 50] HU

tissue). This results in a bilateral filter, where the range similarity is calculated using the temporal MIP M instead of the filtered volume itself. Such kind of approach, where a different image is used to calculate the range similarity, is known as joint bilateral filtering (JBF) [12] in the image processing community and has the same computational effort as standard $3 \mathrm{D}$ bilateral filtering. The filtered weight volume $W_{j}^{\mathrm{JBF}}$ is calculated from $W_{j}$ by:

$$
W_{j}^{\mathrm{JBF}}(\mathbf{v})=k^{-1}(\mathbf{v}) \sum_{\mathbf{v}^{\prime} \in N_{\mathbf{v}}} W_{j}(\mathbf{v}) c\left(\mathbf{v}, \mathbf{v}^{\prime}\right) s\left(\mathbf{M}(\mathbf{v}), \mathbf{M}\left(\mathbf{v}^{\prime}\right)\right),
$$

$$
\begin{aligned}
s\left(\mathbf{M}(\mathbf{v}), \mathbf{M}\left(\mathbf{v}^{\prime}\right)\right) & =\exp \left(-\left(\mathbf{M}(\mathbf{v})-\mathbf{M}\left(\mathbf{v}^{\prime}\right)\right)^{2} / \sigma_{\mathrm{R}}^{2}\right) \\
c\left(\mathbf{v}, \mathbf{v}^{\prime}\right) & =\exp \left(-\left\|\mathbf{v}-\mathbf{v}^{\prime}\right\|_{2}^{2} / \sigma_{\mathrm{D}}^{2}\right) \\
k(\mathbf{v}) & =\sum_{\mathbf{v}^{\prime} \in N_{\mathbf{v}}} c\left(\mathbf{v}, \mathbf{v}^{\prime}\right) s\left(\mathbf{M}(\mathbf{v}), \mathbf{M}\left(\mathbf{v}^{\prime}\right)\right) .
\end{aligned}
$$

Each voxel $\mathbf{v}$ of the filtered volume $W_{j}^{\mathrm{JBF}}$ is a combination of voxels from the original volume $W_{j}$ belonging to neighborhood $N_{\mathbf{v}}$ weighted with the MIP similarity $s$ and the spatial closeness $c$ and normalized by dividing with the sum of all weights $k$. Figure $4 \mathrm{~b}$ shows the MIPs of the initial FDK reconstruction before and after denoising using the JBF approach, where the initial noisy MIP is used as M.

\section{DIR-JBF Algorithm}

Figure 5 shows a flow chart of the complete DIR-JBF algorithm. At first the baseline projections with the static anatomic structures are subtracted from the contrast enhanced projections. Then all rotations are reconstructed using the FDK algorithm with a sharp filter kernel $\left(\sigma_{\mathrm{K}}=0.25\right.$ pixel). In the next step an initial MIP volume $\mathrm{M}$ is calculated from the reconstructed rotations, all initial volumes are denoised using JBF and an updated $\mathrm{M}$ is computed from the denoised reconstructions. The vessel mask in volume space is created by thresholding $\mathrm{M}$ and the vessel masks for all projections of the forward and backward rotations are computed by forward projecting the volume vessel mask using a maximum intensity forward projector. After initializing the weight volumes from

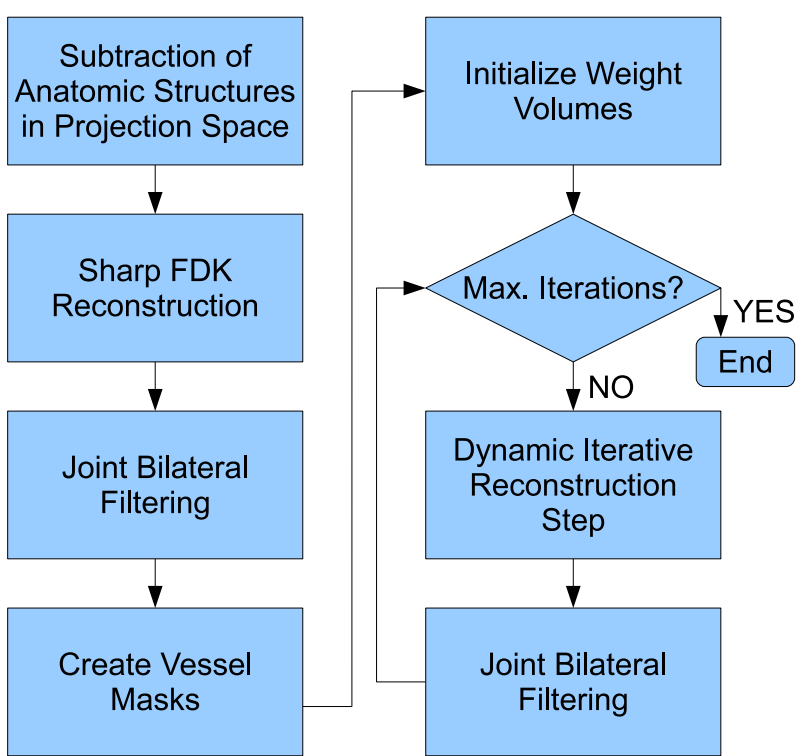

Figure 5: Algorithm overview

\begin{tabular}{c|c} 
Parameter & Value \\
\hline \hline \# Iterations & 6 \\
\hline$\beta$ & $0.6 / N_{\text {proj }}$ \\
\hline$\sigma_{\mathrm{D}}$ & $1 \mathrm{~mm}$ \\
\hline$\tau_{\mathrm{MIP}}$ & $55 \mathrm{HU}$
\end{tabular}

Table II: Parameters DIR-JBF algorithm

the denoised FDK reconstructions a fixed number of iterations is performed. Each iteration consists of a DIR step to achieve data consistency between the weight volumes and the measured projection data followed by denosing of all weight volumes with JBF.

\section{Materials \& Methods}

\section{A. Algorithm Parameters}

The DIR-JBF algorithm parameters used for the experiments are shown in Table II. If one parameter is varied for one experiment, it is stated explicitly in the experiment description.

\section{B. Cylinder Phantom}

For tentative evaluation of the DIR algorithm, a simple dynamic phantom consisting of cylindrical structures describing typical time curves occurring inside a stroke affected brain is used. The MIP of one slice of this phantom is shown in Figure 6a. The cylinders were placed around the origin of a volume of size $256 \times 256 \times 32$ with isotropic voxel spacing of $1 \mathrm{~mm}$ and had a length of $16 \mathrm{~mm}$ in $\mathrm{z}$ direction. The bright white structures describe a real measured arterial input function (AIFs) from clinical PCT (black curve in Figure 7). The light gray, gray and dark gray structures describe TACs in healthy tissue, tissue with reduced perfusion and tissue with severely reduced perfusion, respectively. The tissue TACs were created as described in [13] by convolution of the AIF with a residual function with exponential decay. The perfusion parameters for the different tissue classes were set for $\mathrm{CBF}$ to $53,16,2.5 \mathrm{ml} / 100 \mathrm{ml} / \mathrm{min}$ and for $\mathrm{CBV}$ to 
$3.3,3,0.71 \mathrm{ml} / 100 \mathrm{ml}$ for healthy tissue, tissue with reduced and severely reduced perfusion, respectively. Due to the pauses in the acquisition protocol the quality of the reconstructed TACs depends on the temporal shift of the simulated TACs. Therefore the TACs of every cylinder were computed from an AIF with different temporal shift. The rise of the AIF was varied from $3.5 \mathrm{~s}$ to $7.5 \mathrm{~s}$ after acquisition start with an equal increase of $0.5 \mathrm{~s}$ between adjacent cylinders. The dynamic $\mathrm{C}$-arm projection data was created by forward projecting the 4D phantom according to the acquisition protocol and Poissondistributed noise was added to the projections assuming an emitted X-ray density of $2.1 \cdot 10^{5}$ photons per $\mathrm{mm}^{2}$ at the detector.

\section{Dynamic Brain Phantom}

Classical digital CT phantoms usually consist of homogeneous structures. This highly favors reconstruction algorithms exploiting homogeneity like algorithms using compressed sensing based regularizers (e.g. TV minimization) and also algorithms using regularization by bilateral filtering. Thus, simple extensions to 4D dynamic phantoms do not allow for an authentic evaluation. We adopted the dynamic head phantom from [13], which was originally used for evaluation of perfusion parameter calculation methods, to create an appropriate phantom for evaluating the reconstruction algorithm. Similarly to what is proposed in [13], we segmented brain MRI scans from a human volunteer into white and gray matter, cerebrospinal fluid (CSF), and arteries. White/gray matter and CSF segmentation was done from T1 weighted MRI data using the Freesurfer software [20], [21], [22]. Arteries were segmented from a time-of-flight acquisition by thresholding and manual post-processing. The segmentations were combined into a volume consisting of 150 slices with $256 \times 256$ voxels of isotropic size $1 \mathrm{~mm}$. Inside the volume two different tissue classes were annotated using ellipsoid ROIs: tissue with reduced CBF (2 ROIs, altogether $13197 \mathrm{~mm}^{3}$ ) and tissue with severely reduced CBF and CBV (2 ROIs, altogether $5761 \mathrm{~mm}^{3}$ ). Tissue that was not annotated was simulated as healthy tissue. Different perfusion parameters were assigned to the annotated ROIs as shown in Table III. To further reduce the sparsity of the brain phantom, the MR data was used to vary the perfusion parameters. The parameters were varied according to the intervals shown in Table III. Details of this variation are provided at the phantom web page [14]. The AIF and the tissue TACs were simulated similarly as for the cylinder phantom. To incorporate the anatomic tissue structures into the phantom, appropriate constant HU values were added to the TACs as described in [13]. Noisy projection data was created similarly as for the cylinder phantom.

\section{Canine Study}

The DIR-JBF algorithm was also evaluated using data from an animal study, where an ischemic stroke was induced in a healthy canine under an institutionally approved protocol. Four hours after stroke creation, PCT was acquired and immediately followed by a PCCT acquisition using the same protocol parameters as used for the simulation. The contrast injection

\begin{tabular}{c|c} 
contrast medium & $370 \mathrm{mgI} / \mathrm{ml}$ \\
\hline injection type & intravenous \\
\hline injection rate & $2.0 \mathrm{ml} / \mathrm{s}$ \\
\hline total contrast volume & $28 \mathrm{ml}$ \\
\hline total saline chase volume & $10 \mathrm{ml}$ \\
\hline X-ray delay & $5 \mathrm{~s}$
\end{tabular}

Table IV: Canine study injection protocol

protocol used for both modalities is shown in Table IV. For the real data experiments $\tau_{\mathrm{MIP}}$ was set to $155 \mathrm{HU}$ and $\sigma_{\mathrm{R}}$ was set to $5 \cdot 10^{-4}$. For the FDK reconstruction the static anatomical structures were subtracted in volume and not in projection space and the motion compensation was carried out in the $3 \mathrm{D}$ volume space using a $3 \mathrm{D}-3 \mathrm{D}$ rigid registration based on mutual information [23].

\section{E. Perfusion Parameter Calculation}

To calculate the perfusion parameters, the reconstructed TACs were resampled to a temporal resolution of $1 \mathrm{~s}$ by linear interpolation. A TAC inside the internal carotid artery was selected as AIF in the brain phantom data and a TAC inside the basilar artery was selected as AIF in the canine study data. The perfusion parameters were calculated with our in-house perfusion analysis software using a deconvolution approach based on indicator-dilution theory [24], [25], [26]. Before deconvolution the TAC samples were filtered slice wise in spatial dimension with a 2D Gauss kernel of variance $\sigma^{2}=1 \mathrm{~mm}^{2}$. For quantitative evaluation of the simulation results the root mean square error (RMSE) over time between the reconstructed and the ground truth time curves of the AIF and inside the tissue was computed. To compare the resulting perfusion maps, the Pearson correlation (PC) between maps created from the reconstructed TACs and maps created from the ground truth TACs was computed. The PC was calculated using all voxels of brain slices with stroke annotation belonging to the brain tissue, voxels in and close to vascular structures were excluded. For quantitative evaluation of the canine study perfusion maps we applied the automated ROI analysis with vascular pixel elimination discussed in [3] to calculate the PC between the 8 slices PCT and the coregistered PCCT maps.

\section{RESULTS}

\section{A. Cylinder Phantom}

Figure 6 shows MIP slices of the reconstructed cylinder phantom for FDK reconstruction with $\sigma_{\mathrm{K}}=1$ and DIR-JBF reconstruction with $\sigma_{\mathrm{R}}=10^{-4}$. Examples of reconstructed AIFs from the cylinder phantom using FDK and DIR-JBF algorithm resampled to $1 \mathrm{~s}$ temporal resolution, as used for the perfusion parameter calculation, are shown in Figure 7. Table V shows the RMSE of the reconstructed TACs for the AIFs and the different tissue classes.

\section{B. Dynamic Brain Phantom}

The quantitative comparison between FDK and DIR-JBF is shown in Table VI for different parameter selections of $\sigma_{\mathrm{K}}$ and 


\begin{tabular}{c|c|c|c|c|c|c} 
& \multicolumn{2}{|c|}{ Healthy } & \multicolumn{2}{c|}{ Reduced CBF } & \multicolumn{2}{c}{ Reduced CBF/CBV } \\
& WM & GM & WM & GM & WM & GM \\
\hline CBF $[\mathrm{ml} / 100 \mathrm{ml} / \mathrm{min}]$ & $25 \pm 14$ & $53 \pm 14$ & $7.5 \pm 4.25$ & $16 \pm 4.25$ & $2.5 \pm 1.4$ & $5.3 \pm 1.4$ \\
CBV [ml/100 ml] & $1.9 \pm 0.9$ & $3.3 \pm 0.4$ & $1.7 \pm 0.9$ & $3 \pm 0.7$ & $0.42 \pm 0.2$ & $0.71 \pm 0.12$ \\
MTT [s] & $4.6 \pm 0.7$ & $3.7 \pm 0.7$ & $14 \pm 0.75$ & $11 \pm 0.75$ & $10 \pm 1$ & $8 \pm 1$
\end{tabular}

Table III: Perfusion parameters $(\mathrm{WM}=$ white matter, $\mathrm{GM}=$ gray matter)

\begin{tabular}{c|c|c|c|c} 
Algorithm & \multicolumn{2}{|c}{ FDK } & \multicolumn{2}{c}{ DIR-JBF } \\
\hline Parameter & $\sigma_{\mathrm{K}}=0.25$ & $\sigma_{\mathrm{K}}=1$ & $\sigma_{\mathrm{R}}=10^{-4}$ & $\sigma_{\mathrm{R}}=10^{-5}$ \\
\hline \hline RMSE AIFs [HU] & 55.0 & 89.7 & 25.0 & 35.1 \\
\hline RMSE Tissue Healthy [HU] & 16.4 & 15.9 & 4.4 & 7.1 \\
\hline RMSE Tissue Reduced Perfusion [HU] & 13.6 & 5.0 & 2.9 & 3.0 \\
\hline RMSE Tissue Severely Reduced Perfusion [HU] & 13.4 & 5.4 & 2.3 & 2.1
\end{tabular}

Table V: Quantitative results of the cylinder phantom study showing the root mean square error (RMSE) of the reconstructed time attenuation curves using the FDK and the DIR-JBF approach.

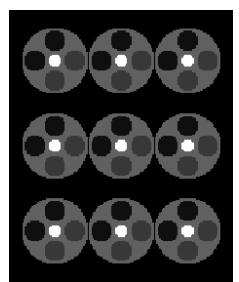

(a) Ground truth

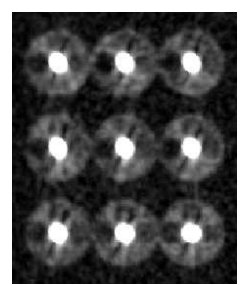

(b) FDK

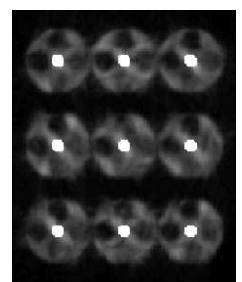

(c) DIR-JBF
Figure 6: MIP cylinder phantom [0 50] HU

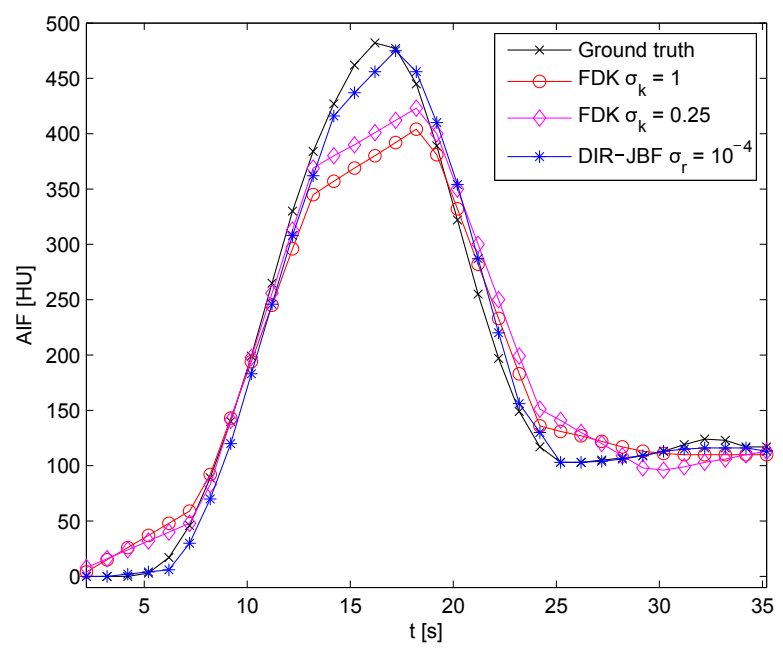

Figure 7: Example AIF from cylinder phantom

$\sigma_{\mathrm{R}}$. Perfusion maps calculated from the reconstructed TACs are shown in Figure 8 for FDK reconstruction with $\sigma_{\mathrm{K}}=1.25$ and DIR-JBF reconstruction with $\sigma_{\mathrm{R}}=1.25 \cdot 10^{-4}$. The TACs of the brain phantom were reconstructed inside a $256 \times 256 \times$ 86 volume with isotropic voxel size $1 \mathrm{~mm}$ covering all brain phantom slices with stroke annotation.

\section{Canine Study}

Figure 9 shows a side-by-side comparison of co-registered PCT and PCCT CBF maps reconstructed with the DIR-JBF and FDK algorithms. Comparing FDK to DIR-JBF reconstruction the PC increased from 0.50 to 0.62 for the CBV maps and from 0.61 to 0.73 for the CBF maps.

\section{DISCUSSION}

The MIPs of the reconstructed cylinder phantom shown in Figure 6 illustrate two advantages of the DIR-JBF algorithm compared to FDK reconstruction. The artifacts around the high contrast artery structures caused by the time-varying attenuation values are reduced in the DIR-JBF result (for a detailed analysis of these artifacts in filtered backprojection reconstruction see [27]). Furthermore the JBF regularization avoids the smoothing of the artery structures into the encircling tissue like in the FDK result. The example AIFs from the cylinder phantom reconstruction in Figure 7 show how the dynamic reconstruction can improve the temporal resolution and avoid the underestimation of the peaks of the TACs compared to FDK reconstruction. Also the quantitative results in Table $\mathrm{V}$ show that the RMSEs of the reconstructed TACs are improved. The perfusion maps created from the brain perfusion data in Figure 8 show that the DIR-JBF perfusion maps compared to the FDK maps are smoother, the stroke affected areas are mostly better separated from the healthy tissue and the vascular structures, which are visible as the red structures, are not blurred into the brain tissue. This also holds for the blood flow maps of the canine study shown in Figure 9. Furthermore a perceptible increase of the PC to the co-registered PCT maps for DIR-JBF compared to FDK reconstruction is observed for the canine $\mathrm{CBF}$ and $\mathrm{CBV}$ maps (Subsection V-C).

\section{CONCLUSIONS}

We introduced a novel dynamic iterative reconstruction (DIR) algorithm with a denoising strategy based on joint bilateral filtering (JBF). The computational efficiency of our algorithm is better compared to existing approaches due to the use of linear spline functions as basis for the TACs. The JBF provides a computational fast, stable and expedient regularization, which is also easy to implement. The results show that the DIR-JBF algorithm has the potential to provide adequate reconstructions of TACs and perfusion maps from slowly rotating $\mathrm{C}$-arm acquisitions. Compared to the FDK algorithm the reconstructed TACs are more accurate both for the cylinder phantom and realistic brain phantom. Also the visual impression and the correlation of the reconstructed brain maps to the ground truth are improved. The PC values 


\begin{tabular}{c|c|c|c|c|c|c|c|c} 
Algorithm & \multicolumn{4}{|c|}{ FDK } & \multicolumn{3}{c}{ DIR-JBF } \\
\hline Parameter & $\sigma_{\mathrm{K}}=0.25$ & $\sigma_{\mathrm{K}}=1$ & $\sigma_{\mathrm{K}}=1.25$ & $\sigma_{\mathrm{K}}=1.5$ & $\sigma_{\mathrm{R}}=1.25 \cdot 10^{-4}$ & $\sigma_{\mathrm{R}}=2.5 \cdot 10^{-4}$ & $\sigma_{\mathrm{R}}=5 \cdot 10^{-4}$ & $\sigma_{\mathrm{R}}=10^{-3}$ \\
\hline \hline RMSE AIF [HU] & 48.00 & 117.03 & 135.05 & 151.57 & 24.17 & 24.12 & 26.10 & 26.61 \\
RMSE Tissue [HU] & 20.63 & 3.48 & 2.74 & 2.35 & 2.16 & 2.11 & 2.09 & 2.10 \\
\hline PC CBF & 0.65 & 0.82 & 0.81 & 0.78 & 0.87 & 0.87 & 0.87 & 0.87 \\
PC CBV & 0.53 & 0.78 & 0.78 & 0.76 & 0.83 & 0.82 & 0.82 & 0.81
\end{tabular}

Table VI: Quantitative results of the brain phantom showing the root mean square error (RMSE) of the reconstructed time attenuation curves and the Pearson correlation (PC) of the CBF and CBV maps to the reference maps using the FDK and the DIR-JBF approach.
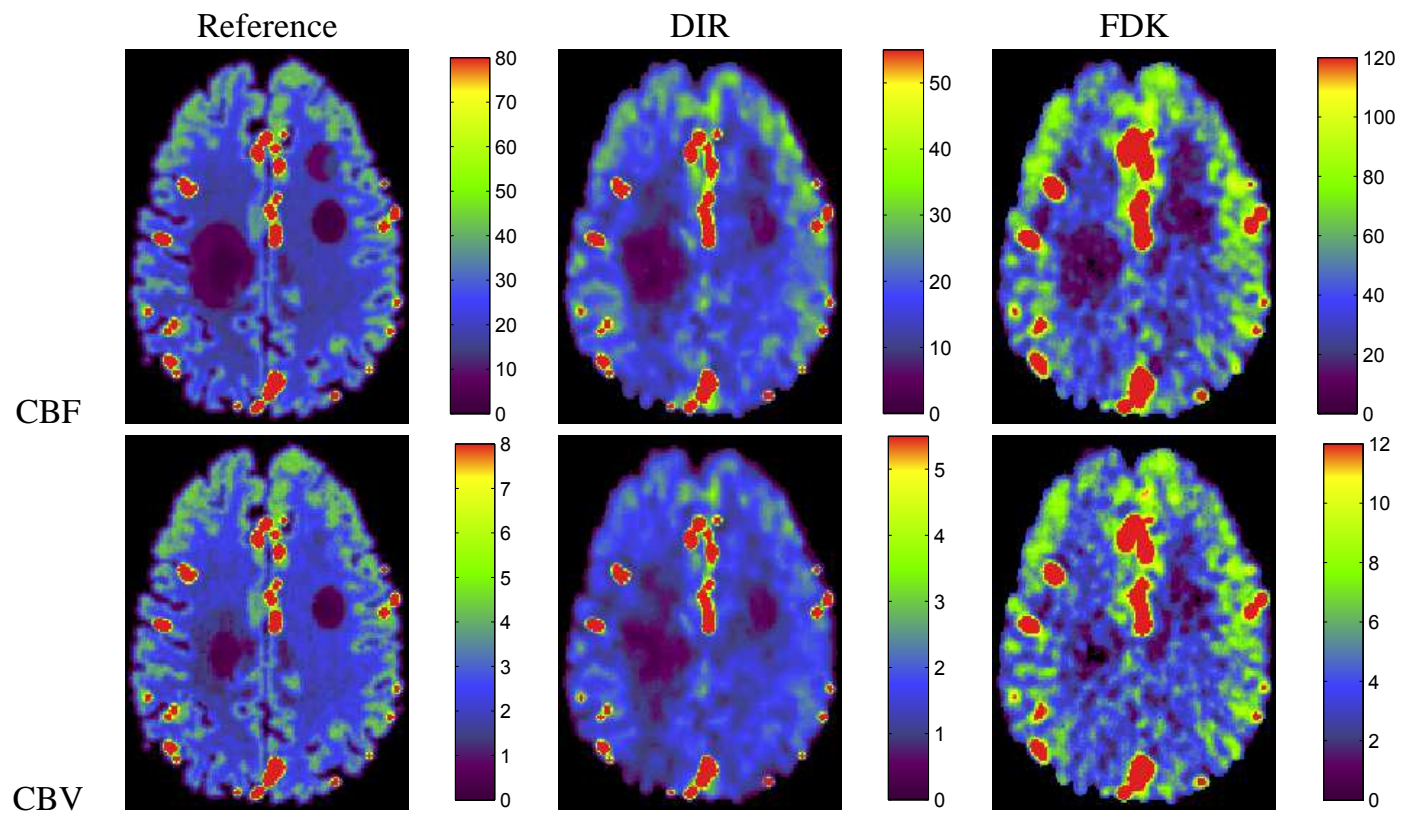

Figure 8: Digital brain phantom CBF (units: $\mathrm{ml} / 100 \mathrm{~g} / \mathrm{min}$ ) and CBV (units: $\mathrm{ml} / 100 \mathrm{~g}$ ) perfusion maps
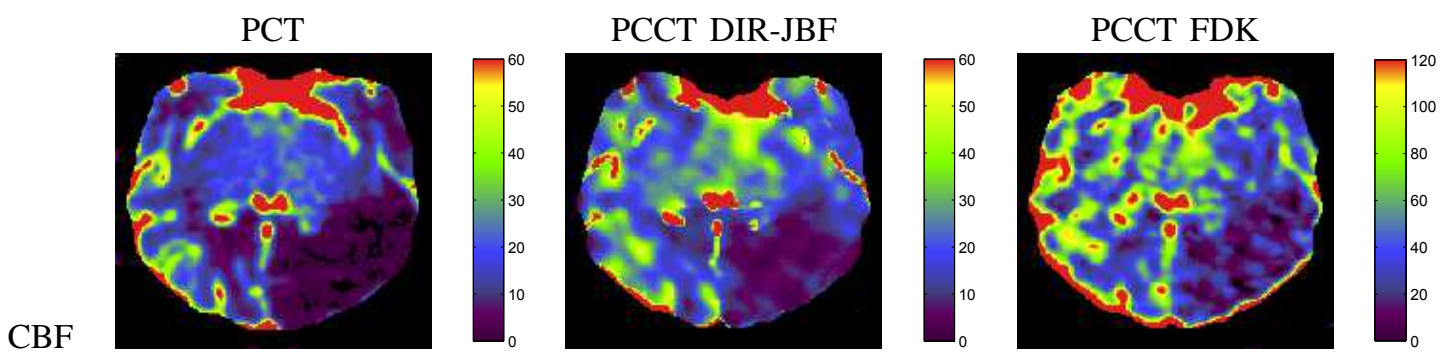

Figure 9: Canine study CBF maps (units: $\mathrm{ml} / 100 \mathrm{~g} / \mathrm{min}$ )

of the canine PCCT perfusion maps to the PCT scan are increased as well. An interesting direction of future research is the comparison to the use of other basis functions like cubic splines, which provide a more realistic description of the smooth TACs and may further improve results on the cost of higher computation time. Furthermore, the subtraction of the static structures in projection space is a potential problem for the clinical application of this algorithm. Patient movement between the acquisitions can lead to sever problems, since a correction of 3D motion in the 2D projection images is difficult due to ambiguity. Further research on how to detect and correct residual structures caused by inaccurate projection subtraction is necessary.

\section{ACKNOWLEDGMENT}

The authors thank Dr. Charles M. Strother and his research team at the Department of Radiology, University of Wisconsin-Madison, USA, for providing the animal study data.

\section{REFERENCES}

[1] R. G. González, J. A. Hirsch, M. H. Lev, P. W. Schaefer, and L. H. Schwamm, Eds., Acute Ischemic Stroke: Imaging and Intervention, 1st ed. Berlin, Germany: Springer, 2005.

[2] A. Fieselmann, "Interventional perfusion imaging using C-arm computed tomography: Algorithms and clinical evaluation," Ph.D. dissertation, Friedrich-Alexander University of Erlangen-Nuremberg, Germany, 2012. 
[3] A. Fieselmann, A. Ganguly, Y. Deuerling-Zheng, M. Zellerhoff, C. Rohkohl, J. Boese, J. Hornegger, and R. Fahrig, "Interventional 4D C-arm CT perfusion imaging using interleaved scanning and partial reconstruction interpolation," IEEE Trans Med Imaging, vol. 31, no. 4, pp. $892-906,2012$.

[4] S. Serowy, O. Gurvit, M. Skalej, and G. Rose, "Jacobi-like solution to the model based tomographic x-ray perfusion imaging," in 2007 IEEE Nuclear Science Symposium Conference Record, vol. 4, 2007, pp. 30853088.

[5] C. Neukirchen, M. Giordano, and S. Wiesner, "An iterative method for tomographic x-ray perfusion estimation in a decomposition model-based approach," Medical Physics, vol. 37, no. 12, pp. 6125-6141, 2010.

[6] A. Kak and M. Slaney, Principles of computerized tomographic imaging. Piscataway, NJ: IEEE Service Center, 1988.

[7] C. Neukirchen, "An extended temporal interpolation approach for dynamic object reconstruction," in Proceedings 11th Fully 3D, 2011, pp. 379-382.

[8] L. Feldkamp, L. Davis, and J. Kress, "Practical cone-beam algorithm," Journal of the Optical Society of America A, vol. 1, no. 6, pp. 612-619, 1984.

[9] E. Y. Sidky and X. Pan, "Image reconstruction in circular cone-beam computed tomography by constrained, total-variation minimization," Physics in Medicine and Biology, vol. 53, no. 17, pp. 4777-4807, 2008.

[10] X. Jia, B. Dong, Y. Lou, and S. B. Jiang, "GPU-based iterative conebeam CT reconstruction using tight frame regularization," Physics in Medicine and Biology, vol. 56, no. 13, pp. 3787-3807, 2011.

[11] C. Tomasi and R. Manduchi, "Bilateral filtering for gray and color images," in Proc. 6th IEEE ICCV, 1998, pp. 839-846.

[12] G. Petschnigg, M. Agrawala, H. Hoppe, R. Szeliski, M. Cohen, and K. Toyama, "Digital photography with flash and no-flash image pairs," in Proc. ACM SIGGRAPH, 2004, pp. 664-672.

[13] A. J. Riordan, M. Prokop, M. A. Viergever, J. W. Dankbaar, E. J. Smit, and H. W. A. M. de Jong, "Validation of CT brain perfusion methods using a realistic dynamic head phantom," Medical Physics, vol. 38, no. 6, pp. 3212-3221, 2011.

[14] Digital brain perfusion phantom. http://www5.cs.fau.de/data/.

[15] D. L. Parker, "Optimal short scan convolution reconstruction for fan beam CT," Medical Physics, vol. 9, no. 2, pp. 254-257, 1982.

[16] L. Shepp and B. Logan, "The Fourier reconstruction of a head section," IEEE Trans Nuclear Science, vol. 21, pp. 21-43, 1974.

[17] B. Keck, H. Hofmann, H. Scherl, M. Kowarschik, and J. Hornegger, "GPU-accelerated SART reconstruction using the CUDA programming environment," in Proc. SPIE Med. Img.: Physics of Medical Imaging, vol. 7258, 2009, pp. 72 582B-1.

[18] V. Aurich and J. Weule, "Non-linear Gaussian filters performing edge preserving diffusion," Proc. DAGM-Symposium Mustererkennung, vol. 17 , pp. 538-545, 1995

[19] A. M. Mendrik, E. Vonken, B. van Ginneken, H. W. de Jong, A. Riordan, T. van Seeters, E. J. Smit, M. A. Viergever, and M. Prokop, "TIPS bilateral noise reduction in $4 \mathrm{D}$ CT perfusion scans produces high-quality cerebral blood flow maps," Physics in Medicine and Biology, vol. 56 , no. 13 , pp. 3857-3872, 2011.

[20] Freesurfer image analysis suite. http://surfer.nmr.mgh.harvard.edu/.

[21] A. M. Dale, B. Fischl, and M. I. Sereno, "Cortical surface-based analysis: I. Segmentation and surface reconstruction," NeuroImage, vol. 9, no. 2, pp. 179-194, 1999.

[22] B. Fischl, A. Liu, and A. M. Dale, "Automated manifold surgery: Constructing geometrically accurate and topologically correct models of the human cerebral cortex," IEEE Trans Med Imaging, vol. 20, pp. 70-80, 2001.

[23] P. Viola and W. M. Wells III, "Alignment by maximization of mutual information," International Journal of Computer Vision, vol. 24, no. 2, pp. 137-154, 1997.

[24] A. Fieselmann, M. Kowarschik, A. Ganguly, J. Hornegger, and R. Fahrig, "Deconvolution-based CT and MR brain perfusion measurement: Theoretical model revisited and practical implementation details," International Journal of Biomedical Imaging, 2011, article ID 467563.

[25] L. Østergaard, R. M. Weisskoff, C. Chesler, David A. Gyldensted, and B. R. Rosen, "High resolution measurement of cerebral blood flow using intravascular tracer bolus passages. Part I: Mathematical approach and statistical analysis," Magnetic Resonance in Medicine, vol. 35, pp. 715725, 1996.

[26] L. Østergaard, A. G. Sorensen, K. K. Kwong, R. M. Weisskoff, C. Gyldensted, and B. R. Rosen, "High resolution measurement of cerebral blood flow using intravascular tracer bolus passages. Part II: Experimental comparison and preliminary results," Magnetic Resonance in Medicine, vol. 36, pp. 726-736, 1996.
[27] A. Fieselmann, F. Dennerlein, Y. Deuerling-Zheng, J. Boese, R. Fahrig, and J. Hornegger, "A model for filtered backprojection reconstruction artifacts due to time-varying attenuation values in perfusion C-arm CT," Physics in Medicine and Biology, vol. 56, no. 12, pp. 3701-3717, 2011. 